\title{
CRESCIMENTO E SOBREVIVÊNCIA DE MUDAS DE EUCALIPTO SOB DOSES DE BORO CULTIVADAS EM CONDIÇÕES DE VIVEIRO E DE CAMPO ${ }^{1}$
}

\author{
Development and survival by eucalypts seedlings under boron rates in field and nursery conditions
}

\author{
Ronaldo Luiz Vaz de Arruda Silveira ${ }^{2}$, Adônis Moreira ${ }^{3}$, Edson Namita Higashi ${ }^{2}$
}

\begin{abstract}
RESUMO
Conduziu-se este trabalho, em condições de viveiro e de campo para avaliar o efeito do boro sobre o crescimento em altura e diâmetro e a sobrevivência de mudas de eucalipto. O delineamento experimental utilizado foi o de blocos casualizados em esquema fatorial 5 x 2: cinco doses de boro $\left(0,0,325,1,3,5,2\right.$ e 20,8 $\left.\mathrm{mg} \mathrm{L}^{-1}\right)$ na forma de octaborato e duas espécies de eucalipto (Eucalyptus grandis W. Hill ex Maiden e Eucalyptus saligna Sm), com cinco repetições. Pelos resultados, verifica-se que as mudas cultivadas com 5,2 e $20,8 \mathrm{mg} \mathrm{L}^{-1}$ de B apresentam redução significativa no crescimento em altura e no diâmetro do caule. Nas condições de campo, a aplicação de até $20,8 \mathrm{mg} \mathrm{L}^{-1}$ não afeta a sobrevivência das mudas das duas espécies de eucalipto. Observou que, nas condições de viveiro, o E. saligna é mais sensível à fitotoxidez de boro do que o $E$. grandis.
\end{abstract}

Termos para indexação: Manejo, altura, diâmetro do caule, Eucalyptus grandis, Eucalyptus saligna.

\begin{abstract}
The objective this work carried out under nursery and field conditions was determine the development and survival by eucalypts seedlings under boron rates. The randomized blocks experimental design in a $5 \times 2$ factorial scheme was used: five rates of boron $\left(0,0.325,1.3,5.2\right.$ and $\left.20.8 \mathrm{mg} \mathrm{L}^{-1}\right)$ and two species (Eucalyptus grandis W. Hill ex Maiden and Eucalyptus saligna $\mathrm{Sm}$ ), with five replicates. The results showed that in two conditions, the eucalypts seedlings grown with 5.2 and $20.8 \mathrm{mg} \mathrm{L}^{-1}$ of boron had significative reduction in diameter and height. The application at $20.8 \mathrm{mg} \mathrm{L}^{-1}$ of boron did not affect the two eucalypts species survival in the field. The Eucalyptus saligna was more sensible than Eucalyptus grandis to boron rates under nursery conditions.
\end{abstract}

Index terms: Management, height, stem diameter, Eucalyptus grandis, Eucalyptus saligna.

(Recebido para publicação em 27 de janeiro de 2003 e aprovado em 21 de maio de 2003)

\section{INTRODUÇÃO}

O elevado número de espécies e clones confere ao eucalipto grande possibilidade de expansão geográfica e econômica. Estima-se, no Brasil, que a área cultivada apenas para o setor de papel e celulose com espécies do gênero Eucalyptus atinja 1,43 milhão de hectares. Além disso, essas espécies são adaptadas às mais diversas condições de edafoclimáticas, como também a inúmeros tipos de explorações, tais como: produção de dormentes, poste, lenha (carvão), confecção de móveis, extração de óleo essencial, mourões e, principalmente, como citado acima, na extração de celulose para fabricação de papel (INSTITUTO IGUAÇU, 1994).

Além das suas qualidades, sua expansão deveuse, principalmente, ao uso de mudas adequadamente manejadas, provenientes de matrizes de alta produtividade e do uso de fertilizantes e corretivos do solo
(MOREIRA et al., 1999), haja vista que o cultivo do eucalipto se concentra em terras de baixa fertilidade, como os Neossolos e Latossolos (SGARBI et al., 1999).

Nesse contexto, o boro, que atua no crescimento meristemático das plantas (MARSCHNER, 1995; SILVEIRA, 1996), destaca-se como sendo um dos nutrientes que mais limitam o desenvolvimento do eucalipto. Apesar do papel fisiológico desse nutriente ainda não estar totalmente entendido, sabe-se, entretanto, da sua importância na formação da parede celular, mais especificamente na síntese dos seus componentes, como a pectina, a celulose e a lignina (MARSCHNER, 1995; MORAES et al., 2002).

Os sintomas de deficiência e de toxidez desse nutriente estão restritos a sua mobilidade, considerada baixa ou muito limitada no floema. Descobertas recentes, entretanto, demonstram que a mobilidade do B

1. Projeto financiado pela Siderúrgica Barra Mansa, Capão Bonito, Estado de São Paulo.

2. RR Agroflorestal , 13400-970 - Piracicaba, SP. ronaldo@rragroflorestal.com.br e edson@rragroflorestal.com.br.

3. Embrapa Amazônia Ocidental, Caixa Postal 319-69011-970 - Manaus, AM. adonis@cpaa.embrapa.br. Bolsista CNPq. 
não é restrita a todas as espécies e em algumas, como por exemplo, a macieira (HU e BROWN, 1997). Em trabalhos realizados por Salvador et al. (2003) verifica-se que na goiabeira, espécie da mesma família das mirtáceas, o boro apresenta baixa mobilidade no floema.

Yamada (2000) ressaltou que para ocorrer um bom desenvolvimento das plantas se faz necessária a adubação adequada com boro, em razão principalmente, do seu papel no desenvolvimento das raízes, sendo esse essencial para o crescimento da parte aérea e do sistema radicular das plantas.

Objetivou-se com este trabalho avaliar o efeito do boro sobre o crescimento em diâmetro e altura e a sobrevivência de mudas de Eucalyptus grandis W. Hill ex Maiden e Eucalyptus saligna Sm, cultivadas em condições de viveiro e de campo.

\section{MATERIAL E MÉTODOS}

Os experimentos foram conduzidos em condições de viveiro e de campo em área de cultivo da Siderúrgica Barra Mansa, localizada no município de Capão Bonito, Estado de São Paulo.

Empregou-se o delineamento em bloco casualizados em esquema fatorial 5 × 2 : cinco doses de boro $\left(0,0,325,1,30,5,20\right.$ e $\left.20,8 \mathrm{mg} \mathrm{L}^{-1}\right)$ na forma de octaborato (solubor - BQ) e duas espécies de eucalipto (Eucalyptus grandis W. Hill ex Maiden e Eucalyptus saligna $\mathrm{Sm}$ ), com cinco repetições. Independentemente da condição estudada, as plantas receberam adubações parceladas em três vezes. No experimento de viveiro e de campo, as plantas foram adubadas com os seguintes fertilizantes: MAP - 18 $\mathrm{kg}, \mathrm{CaNO}_{3}-27 \mathrm{~kg}, \mathrm{MgSO}_{4}-15 \mathrm{~kg}, \mathrm{KCl}-10 \mathrm{~kg}$, Uréia - $2 \mathrm{~kg}, \mathrm{CuSO}_{4}-200 \mathrm{~g}, \mathrm{FeSO}_{4}-15 \mathrm{~kg}$, $\mathrm{Na}_{2} \mathrm{MoO}_{4}-35 \mathrm{~g}, \mathrm{MnSO}_{4}-3,6 \mathrm{~kg}, \mathrm{ZnSO}_{4}-620 \mathrm{~g}$, diluídos em 100 litros de água. As plantas transplantadas para o campo tiveram uma adubação complementar de $2,4 \mathrm{~kg}$ de $\mathrm{H}_{3} \mathrm{BO}_{3}$ quando estavam na fase de viveiro.

As mudas de eucalipto eram provenientes de microestacas e cultivadas em tubetes de polipropileno com capacidade de $50 \mathrm{~cm}^{3}$ de extrato. No experimento de campo, as mudas foram retiradas do viveiro e transplantadas aos 86 dias em parcelas experimentais (blocos) de $306,25 \mathrm{~m}^{2}$ (espaçamento $2,5 \mathrm{~m} \mathrm{x} \mathrm{2,5} \mathrm{m).}$ Avaliaram-se 25 plantas por parcelas, totalizando 125 plantas por tratamento.
As plantas cultivadas no viveiro foram avaliadas aos 86 dias de idade, ao passo que as de campo, aos 180 dias após o transplantio (266 dias de idade). Mediram-se a altura, o diâmetro (altura do colo nas plantas no viveiro e a $1,30 \mathrm{~m}$ do solo nas plantas no campo - DAP) e a porcentagem de sobrevivência das mudas.

Os resultados foram submetidos à análise de variância (teste $\mathrm{F}$ ), teste de comparação de contraste de médias (Tukey 5\%) e correlações, conforme metodologias descritas por Gomes (1990).

\section{RESULTADOS E DISCUSSÃO}

Os efeitos das doses de boro sobre o crescimento em altura das duas espécies de eucalipto são mostrados na Tabela 1 . Observa-se que, na ausência de B, as plantas apresentaram menor crescimento em altura que as doses 0,325 e 1,30 $\mathrm{mg} \mathrm{L}^{-1}$. Novelino et al. (1982), Silveira et al. (2000) e Maffeis et al. (2000) também constataram que a omissão de boro limita significativamente o crescimento em altura e diâmetro do Eucalipto. Segundo Loué (1993) e Moraes et al. (2002), na deficiência de boro, ocorre redução da síntese de pectina e lignina, tornando mais finas as paredes das células do lenho, o que acarreta num menor crescimento das plantas.

A elevação da disponibilidade de boro acima de $1,30 \mathrm{mg} \mathrm{L}^{-1}$ ocasionou em decréscimo significativo no crescimento em altura, sendo esse resultado mais expressivo na fase de viveiro. Nesse estágio, as mudas apresentaram redução, quando comparada com a testemunha, de $7,14 \%$ e $9,09 \%$ na dose 5,2 $\mathrm{mg} \mathrm{L}^{-1}$ de B e de $28,97 \%$ e $36,36 \%$ na dose $20,8 \mathrm{mg}$ $\mathrm{L}^{-1}$ de $\mathrm{B}$ para o E. grandis e E. saligna, respectivamente.

No campo, a redução no crescimento em altura a partir da dose $1,30 \mathrm{mg} \mathrm{L}^{-1}$ foi menos acentuada que nas condições de viveiro (Tabela 1). Nessa condição, a eficiência na absorção de boro está intimamente relacionada com a presença de óxidos de ferro e alumínio, matéria orgânica, minerais de argila, pH e principalmente água (LOUÉ, 1993). Outro fator que deve ter influenciado a menor resposta das plantas aos tratamentos foi o efeito residual do boro aplicado nas plantas no estágio de viveiro.

Como citado anteriormente, a redução no crescimento em altura na carência de $\mathrm{B}$ deve-se à atuação do nutriente no crescimento meristemático da planta (MARSCHNER, 1995). Nos tratamentos com 
as maiores doses, também foram observadas necroses nas folhas novas e morte da gema apical. Esse resultado confirma os obtidos por Maffeis et al. (2000) com Eucalyptus citriodora Hook, em que a ausência ou excesso de boro, provoca nas plantas a morte da gema apical, com posteriores brotações das gemas axilares, o que resulta na paralisação do crescimento.

$\mathrm{Na}$ comparação entre as duas espécies de eucalipto, foram verificados respostas distintas à fertilização com boro para a variável altura (Tabela 1). No viveiro, o E. saligna apresentou, na média, a maior taxa de crescimento; o inverso ocorreu com as mudas transplantadas para o campo. Silveira et al. (1995) estudaram a absorção de nutrientes por clones de eucalipto e constataram que o E. grandis apresentou maior acúmulo de nutrientes que o E. saligna. Tal resultado é um indicativo da maior eficiência na absorção de nutrientes de uma espécie em relação a outra, tendo como seqüência um maior desenvolvimento das plantas. Dentro das mesmas condições, não houve interação espécies e doses.

Pelos valores de diâmetro (Tabela 2), infere-se que nas duas espécies as plantas cultivadas no viveiro apresentaram redução significativa a partir da dose
$1,30 \mathrm{mg} \mathrm{L}^{-1}$, estando essa variável intimamente relacionada com o crescimento das mudas (Figura 1), o mesmo ocorreu nas condições de campo, corroborando os resultados de Silveira et al. (2000) com Eucalyptus citriodora Hook. Com esse resultado demonstra-se a necessidade de definir um nível adequado de boro para cada espécie de eucalipto, sendo essa inferência mais expressiva quando as mudas foram transplantadas para o campo.

Com essa interação, verifica-se que a carência ou excesso de boro pode acarretar alterações na síntese de lignina (MARSCHNER, 1995; MORAES et al., 2002), com conseqüente morte da gema apical, o que afeta significativamente o desenvolvimento em crescimento das plantas.

Quanto à porcentagem de sobrevivência das mudas de E. grandis e E. saligna (Tabela 3), não foram observadas diferenças significativas nas duas condições estudadas em razão das doses de boro, ficando os valores acima de $95 \%$ nas condições de campo. No viveiro, independentemente dos tratamentos, a taxa de sobrevivência foi de $100 \%$, o que significa que até a dose de $20,8 \mathrm{mg} \mathrm{L}^{-1}$, nas condições estudadas, não ocorre a morte das duas espécies de eucalipto por fitotoxidez de boro.

TABELA 1 - Altura das plantas de Eucalyptus grandis e Eucalyptus saligna em razão das doses de boro cultivadas no viveiro e no campo ${ }^{1}$.

\begin{tabular}{|c|c|c|c|c|}
\hline \multirow{2}{*}{$\begin{array}{c}\text { Doses de boro } \\
\text { mg L }^{-1}\end{array}$} & \multicolumn{2}{|c|}{ Viveiro } & \multicolumn{2}{|c|}{ Campo } \\
\hline & E.grandis & E. saligna & E. grandis & E. saligna \\
\hline 0,0 & $0,28 b$ & $0,33 \mathrm{a}$ & $2,34 \mathrm{~A}$ & $2,26 \mathrm{~A}$ \\
\hline 0,325 & $0,30 \mathrm{~b}$ & $0,36 \mathrm{a}$ & $2,39 \mathrm{~A}$ & $2,34 \mathrm{~A}$ \\
\hline 1,3 & $0,32 \mathrm{a}$ & $0,34 \mathrm{a}$ & $2,36 \mathrm{~A}$ & $2,15 \mathrm{~B}$ \\
\hline 5,2 & $0,26 b$ & $0,30 \mathrm{a}$ & $2,32 \mathrm{~A}$ & $2,11 \mathrm{~B}$ \\
\hline 20,8 & $0,20 \mathrm{a}$ & $0,21 \mathrm{a}$ & $2,18 \mathrm{~A}$ & $2,13 \mathrm{~B}$ \\
\hline Média & 0,27 & 0,31 & 2,32 & 2,20 \\
\hline Teste F & $56,36^{* *}$ & & $36,14 * *$ & \\
\hline C.V. (\%) & 9,53 & & 7,62 & \\
\hline
\end{tabular}

Ciênc. agrotec., Lavras, v. 28, n. 2, p. 366-371, mar./abr., 2004 
TABELA 2 - Diâmetro das plantas de Eucalyptus grandis e Eucalyptus saligna em razão das doses de boro cultivadas no viveiro (altura do colo $-10 \mathrm{~cm}$ ) e no campo (DAP - 1,3 metro) ${ }^{1}$.

\begin{tabular}{|c|c|c|c|c|}
\hline \multirow{2}{*}{$\begin{array}{c}\text { Doses de boro } \\
\qquad \mathrm{mg} \mathrm{L}^{-1}\end{array}$} & \multicolumn{2}{|c|}{ Viveiro } & \multicolumn{2}{|c|}{ Campo } \\
\hline & E.grandis & E. saligna & E. grandis & E. saligna \\
\hline & \multicolumn{4}{|c|}{ _ (milímetros) - } \\
\hline 0,0 & $3,72 \mathrm{a}$ & $3,40 \mathrm{~b}$ & $36,00 \mathrm{~A}$ & $28,45 \mathrm{~B}$ \\
\hline 0,325 & $3,60 \mathrm{a}$ & $3,40 \mathrm{~b}$ & $36,95 \mathrm{~A}$ & $34,60 \mathrm{~A}$ \\
\hline 1,3 & $3,82 \mathrm{a}$ & $3,38 b$ & $35,90 \mathrm{~A}$ & $36,60 \mathrm{~A}$ \\
\hline 5,2 & $3,41 \mathrm{a}$ & $3,31 \mathrm{a}$ & $31,55 \mathrm{~A}$ & $34,72 \mathrm{~A}$ \\
\hline 20,8 & $2,90 \mathrm{a}$ & $2,54 \mathrm{~b}$ & $33,50 \mathrm{~A}$ & $34,35 \mathrm{~A}$ \\
\hline Média & 2,75 & 3,21 & 34,78 & 33,74 \\
\hline Teste F & $65,25 * *$ & & $38,32 * *$ & \\
\hline C.V. $(\%)$ & 14,12 & & 16,85 & \\
\hline
\end{tabular}

** Significância das doses de B nas duas espécies de eucalipto, a $1 \%$ de probabilidade. ${ }^{1}$ Médias seguidas por letras distintas minúsculas (viveiro) e maiúsculas (campo) na mesma dose (linha) diferem entre si a $5 \%$ de probabilidade pelo teste de Tukey.

TABELA 3 - Sobrevivência das plantas de Eucalyptus grandis e Eucalyptus saligna em razão das doses de boro cultivadas no viveiro e no campo ${ }^{1}$.

\begin{tabular}{cccccc}
\hline \multirow{2}{*}{ Doses de boro } & \multicolumn{2}{c}{ Viveiro } & & \multicolumn{2}{c}{ Campo } \\
\cline { 2 - 3 } \cline { 5 - 5 } mg L $^{-1}$ & E.grandis & E. saligna & & E. grandis & E. saligna \\
& $-100 \mathrm{a}$ & $100 \mathrm{a}$ & & $99 \mathrm{~A}$ & $97 \mathrm{~A}$ \\
0,0 & $100 \mathrm{a}$ & $100 \mathrm{a}$ & & $100 \mathrm{~A}$ & $97 \mathrm{~A}$ \\
0,325 & $100 \mathrm{a}$ & $100 \mathrm{a}$ & & $100 \mathrm{~A}$ & $100 \mathrm{~A}$ \\
1,3 & $100 \mathrm{a}$ & $100 \mathrm{a}$ & & $96 \mathrm{~A}$ & $100 \mathrm{~A}$ \\
5,2 & $100 \mathrm{a}$ & $100 \mathrm{a}$ & & $97 \mathrm{~A}$ & $98 \mathrm{~A}$ \\
20,8 & 100 & 100 & & 98 & 98 \\
Média & $0,00^{\mathrm{NS}}$ & & $3,16^{\mathrm{NS}}$ & \\
\hline Teste $\mathrm{F}$ & 0,00 & & 3,30 & \\
C.V. $(\%)$ & & &
\end{tabular}

${ }^{\mathrm{NS}}$ Não-significância das doses de B nas duas espécies a $1 \%$ de probabilidade. ${ }^{1}$ Médias seguidas por letras distintas minúsculas (viveiro) e maiúsculas (campo) na mesma dose (linha) diferem entre si a $5 \%$ de probabilidade pelo teste de Tukey. 

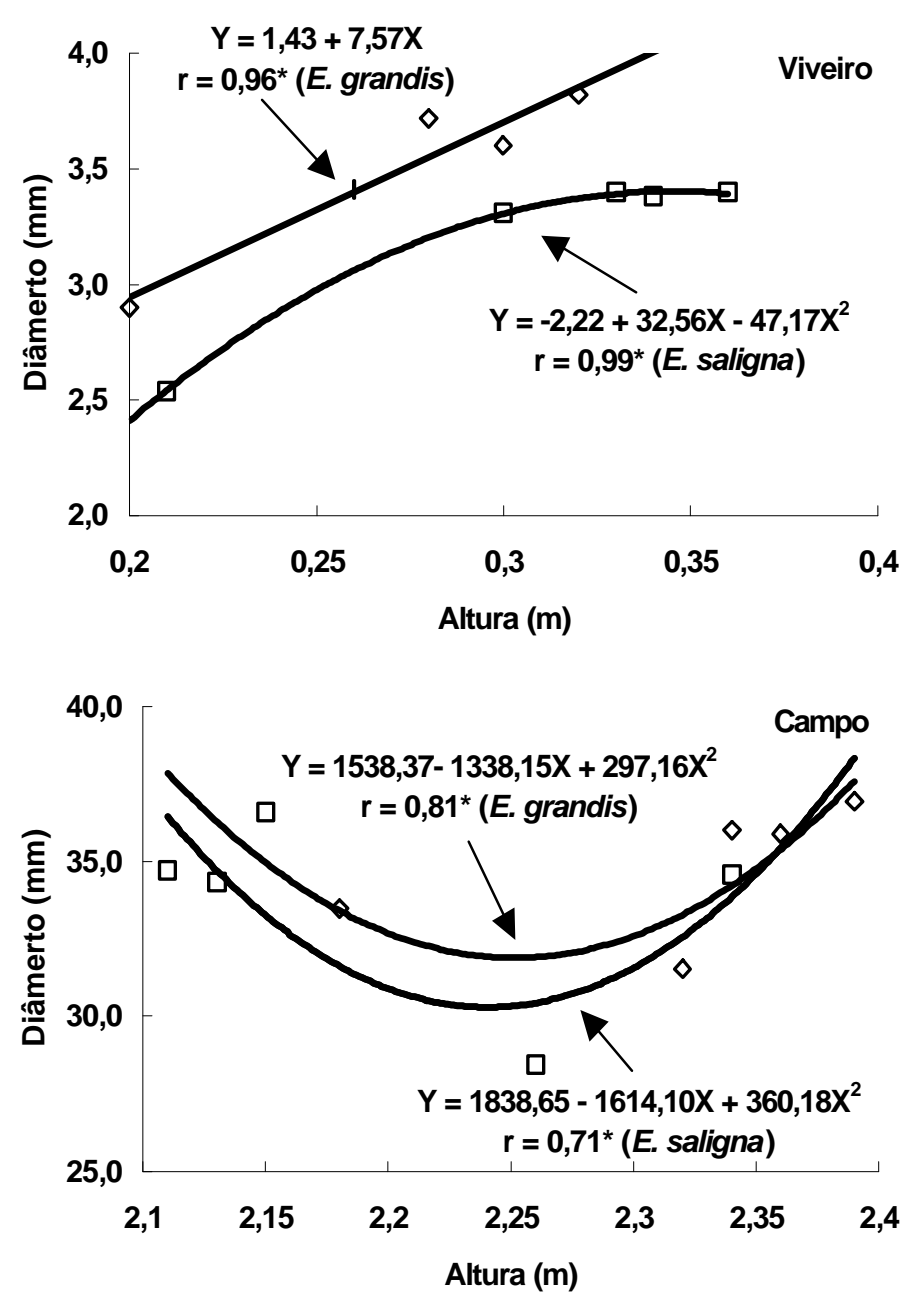

FIGURA 1 - Relação entre a altura e o diâmetro do caule das duas espécies de eucalipto (Eucalyptus grandis e Eucalyptus saligna) nas duas condições estudadas. * Significativo a 5\% de probabilidade pelo teste F.

\section{CONCLUSÕES}

a) As mudas de Eucalyptus saligna W. Hill ex Maiden e Eucalyptus grandis Sm, com até 266 dias de idade, cultivadas com doses iguais ou superiores a 5,2 $\mathrm{mg} \mathrm{L}^{-1}$ apresentam redução significativa no crescimento em altura e no diâmetro do caule.

b) A aplicação de até $20,8 \mathrm{mg} \mathrm{L}^{-1}$ não afeta o pegamento e sobrevivência das mudas de $E$. grandis e E. saligna.

c) No estágio de viveiro, o Eucalyptus saligna é mais sensível à fitotoxidez de boro do que o Eucapyptus grandis.

\section{REFERÊNCIAS BIBLIOGRÁFICAS}

GOMES, F. P. Curso de estatística experimental. Piracicaba: Nobel, 1990.

HU, H.; BROWN, P. H. Absorption of boron by plants roots. In: DELL, B.; BROWN, P. H.; BELL, R. W. (Eds.). Boron in soils and plants: reviews. Dordrecht: Kluwer Academic, 1997. p. 49-58.

INSTITUTO IGUAÇÚ. Áreas de conservação de caráter privado: módulos alternativos e incentivos. In: PESQUISA E PRESERVAÇÃO AMBIENTAL, WORKSHOP, 1994, Rio de Janeiro. Resumos... Rio de Janeiro, 1994. $123 \mathrm{p}$. 
LOUÉ, A. Oligo-éléments em agriculture. Antibes: Nathan, 1993. 577 p.

MAFFEIS, A. R.; SILVEIRA, R. L. V. A.; BRITO, J. O. Reflexos das deficiências de macronutrientes e boro no crescimento de plantas, produção e qualidade de óleo essencial em Eucalyptus citriodora. Scientia Forestalis, Piracicaba, v. 57, p. 87-98, 2000.

MARSCHNER, H. Mineral nutrition of higher plants. London: Academic, 1995. 889 p.

MORAES, L. A. C.; MORAES, V. H. F.; MOREIRA, A. Relação entre flexibilidade do caule de seringueira e a carência de boro. Pesquisa Agropecuária Brasileira, Brasília, v. 37, p. 1431-1436, 2002.

MOREIRA, A.; HIGASHI, E. H.; SILVEIRA, R. L. V. A. Monitoramento nutricional na LWARCEL. Piracicaba: LWARCEL; IPEF; RR Agroflorestal, 1999. $62 \mathrm{p}$.

NOVELINO, J. O.; NEVES, J. C. L.; BARROS, N. F.; NOVAIS, R. F.; MUNIZ, A. S. Efeito de níveis em solução nutritiva no crescimento de mudas de Eucalyptus spp. Revista Árvore, Viçosa, v. 6, p. 45-51, 1982.

SALVADOR, J. O.; MOREIRA, A.; MALAVOLTA, E.; CABRAL, C. P. Influência do boro e do manganês no crescimento e na composição mineral de mudas de goiabeira. Ciência e Agrotecnologia, Lavras, v. 27, p. 325-331, 2003.
SGARBI, F.; SILVEIRA, R L. V. A.; TAKAHASHI, E. N.; CAMARGO, M. A. F. Crescimento e produção de biomassa de clone de Eucalyptus grandis x Eucalyptus urophylla em condições de deficiência de macronutrientes, B e Zn. Scientia Forestalis, Piracicaba, v. 56, p. 69-82, 1999.

SILVEIRA, R. L. V. A. Crescimento e estado nutricional de Eucalyptus citriodora sob doses de boro e sua relação com a agressividade de Brotrysphaeria ribis. 1996. 100 f. Dissertação (Mestrado em Solos e Nutrição de Plantas) - Escola Superior de Agricultura Luiz de Queiroz, Piracicaba, 1996.

SILVEIRA, R. L. V. A.; CAMARGO, M. A. F.; LUCCA, E. F.; LUZ, H. F. Absorção e exportação de macronutrientes pelas brotações de Eucalyptus grandis e Eucalyptus saligna em jardim clonal. In: CONGRESSO BRASILEIRO DE CIÊNCIA DO SOLO, 25., 1995, Viçosa. Resumos Expandidos... Viçosa: SBCS; UFV, 1995. p. 845-847.

SILVEIRA, R. L. V. A.; TAKAHASHI, E. N.; SGARBI, F.; CAMARGO, M. A. F.; MOREIRA, A. Crescimento e estado nutricional de brotações de Eucalyptus citriodora sob doses de boro em solução nutritiva. Scientia Forestalis, Piracicaba, v. 57, p. 53-67, 2000.

YAMADA, T. Boro: será que estamos aplicando a dose suficiente para adequado desenvolvimento das plantas? Informações Agronômicas, Piracicaba, n. 90, p. 1-5, 2000 . 